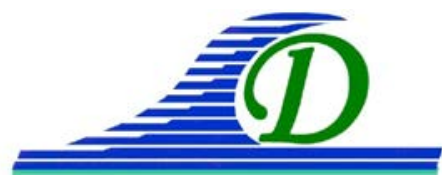

XIII İmes Journées Nationales Génie Côtier - Génie Civil Dunkerque, 2-4 juillet 2014

DOI:10.5150/jngcgc.2014.081 C Editions Paralia CFL

disponible en ligne - http://www.paralia.fr - available online

\title{
Mise en évidence de l'importance de la turbulence ambiante sur les effets d'interaction entre hydroliennes
}

\author{
Youen KERVELLA ${ }^{1}$, Grégory GERMAIN ${ }^{2}$, Benoît GAURIER ${ }^{2}$, \\ Jean-Valéry FACQ ${ }^{2}$, Thomas BACCHETTI ${ }^{2}$
}

\section{OPEN OCEAN, 15 rue Kepler, 29200 Brest, France.}

Youen.kervella@openocean.fr

2. IFREMER, Marine Structures Laboratory,

150 quai Gambetta, BP 699, F-62321 Boulogne-Sur-Mer, France.

\{benoit.gaurier, gregory.germain, jvfacq, thomas.bacchetti\}@ifremer.fr

\section{Résumé :}

Les futures zones d'implantation des parcs hydroliens en mer sont caractérisées par des courants de forte intensité et des taux de turbulence importants. Cette turbulence de l'écoulement est susceptible d'altérer le comportement des hydroliennes, à la fois en termes de production d'énergie mais aussi de fatigue structurelle.

Afin de déterminer l'influence de la turbulence ambiante sur les effets d'interaction entre hydroliennes, des essais expérimentaux ont été effectués dans un bassin à houle et courant, sur des maquettes d'hydroliennes tri-pales à axe horizontal, régulées en vitesse de rotation. Les interactions entre trois hydroliennes placées sur deux rangées (deux hydroliennes sur la rangée amont et une sur la rangée aval, ou inversement) ont été étudiées. Pour ce faire, les performances des machines ont été évaluées à l'aide d'une mesure de couple sur l'arbre de chaque turbine et le sillage de l'ensemble des trois machines à partir de mesures par vélocimétrie laser 2D.

Ces mesures de comportement et de performances ont été effectuées à une vitesse de courant fixée à $0.8 \mathrm{~m} / \mathrm{s}$ avec des taux de turbulence de l'écoulement amont de $3 \%$ et de $15 \%$.

L'impact de ces deux taux de turbulence sur les performances des machines et sur le développement de leurs sillages est donc mis en évidence dans ce papier. Des préconisations pour les futures modélisations numériques seront également établies.

Mots-clés : Énergie marine, Hydroliennes, Turbulence, Effets d'interaction, Essais expérimentaux.

\section{Introduction}

Alors que les premières technologies d'hydroliennes atteignent la maturité en ayant été testées en mer pour leur résistance, la perspective du développement de parcs d'hydrolienne devient réaliste. Il est par conséquent essentiel de mieux comprendre l'impact qu'auront ces parcs d'hydroliennes sur leur environnement, et notamment sur la dynamique des courants marins à l'échelle régionale (BATIFOULIER et al., 2012). Il 


\section{Thème 5 - Énergies et ressources marines}

faut avant tout connaître les interactions fines entre les hydroliennes qui composeront les rangées des futurs parcs (figure 1). Ces interactions, liées aux développements de sillages des hydroliennes et au site d'implantation des parcs, sont fortement influencés par la turbulence ambiante de l'écoulement comme ont commencé à le montrer de récentes études (MYCEK et al., 2014 ; MYERS \& BAHAJ, 2010) à l'aide de mesures expérimentales et de modélisations autour d'une ou de deux hydroliennes (CARLIER et al., 2014).

Ce papier présente l'influence du taux de turbulente de l'écoulement incident sur le développement des sillages, mais également sur les performances intrinsèques des machines, en se basant sur deux configurations tests présentant deux positionnements relatifs entre trois machines réalistes (figure 2).

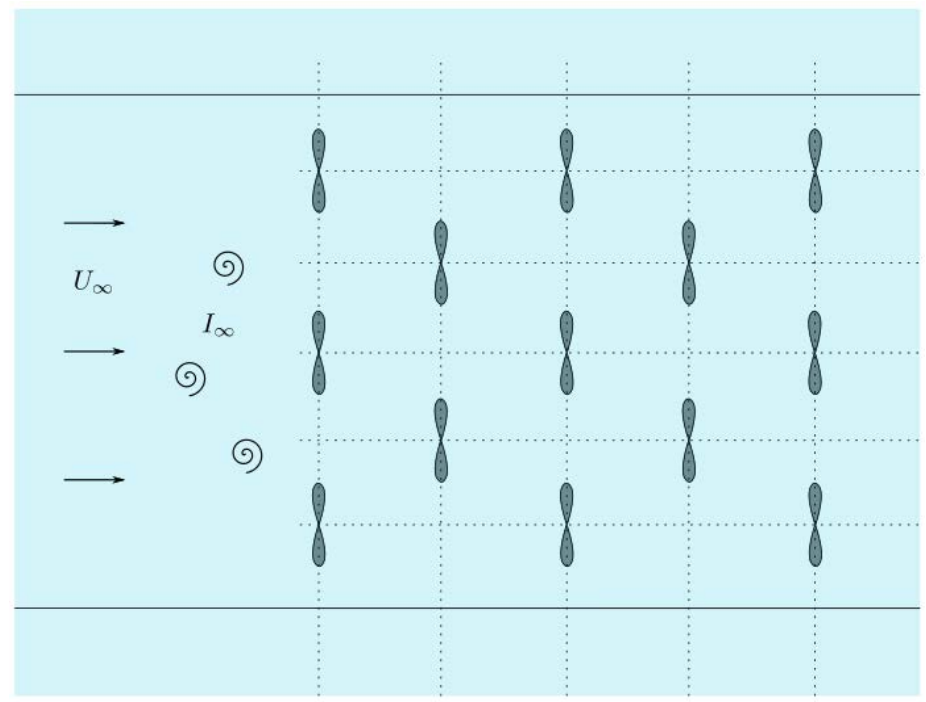

Figure 1. Configuration théorique d'une ferme d'hydrolienne.

\section{Dispositif expérimental}

Le dispositif expérimental utilisé pour étudier le comportement de trois hydroliennes en interaction dans le bassin à houle et à courant de l'IFREMER de Boulogne-sur-Mer est schématisé par la figure 2. Le modèle d'hydrolienne utilisé pour ces essais est une machine tri-pales à axe horizontal. Elle possède un diamètre de $700 \mathrm{~mm}$ et est équipée de pales dont la forme a été optimisée pour des vitesses de courant de 0.6 à $1.2 \mathrm{~m} / \mathrm{s}$, i.e. des nombres de Reynolds basés sur le rayon entre $210 \times 10^{3}$ et $420 \times 10^{3}$. La section d'essais faisant $4 \times 2 \mathrm{~m}^{2}$, le coefficient de blocage est inférieur à $5 \%$ par machine. Ces dernières se trouvant au centre du bassin, elles ne perçoivent aucun gradient de vitesse ni d'intensité turbulente provoqué par la couche limite sur les murs et le fond, ni même d'effet de surface libre.

Les paramètres influençant le comportement de l'hydrolienne que l'on peut contrôler sont: la vitesse moyenne de l'écoulement amont notée $U_{\infty}$; le taux de turbulence 


\section{XIII ${ }^{\text {èmes }}$ Journées Nationales Génie Côtier - Génie Civil \\ Dunkerque, 2-4 juillet 2014}

ambiante, notée $I_{\infty}$ et le Tip Speed Ratio (TSR), qui est défini comme étant le ratio entre la vitesse en bout de pale et la vitesse amont $U_{\infty}$ :

$T S R=\frac{\Omega R}{U_{\infty}}$ avec $R=D / 2$ le rayon de l'hydrolienne et $\Omega$ sa vitesse de rotation.

Les hydroliennes utilisées pour ces travaux sont régulées en vitesses de rotation, la mesure de couple est directe et se mesure à l'aide d'un couplemètre rotatif. Les performances peuvent ainsi être déterminées de manière classique par le calcul des coefficients de puissance $C_{P}$ et de trainée $C_{T}$ qui sont définis par :

$C_{P}=\frac{M_{x} \Omega}{\frac{1}{2} \rho \pi R^{2} U_{\infty}^{3}}$,et: $C_{T}=\frac{F_{X}}{\frac{1}{2} \rho \pi R^{2} U_{\infty}^{2}}$

avec $M_{X}$ le moment en $x$ (orienté dans le sens de l'écoulement amont), $\rho$ la masse volumique du fluide et $F_{X}$ l'effort de traînée mesuré sur toute l'hydrolienne.
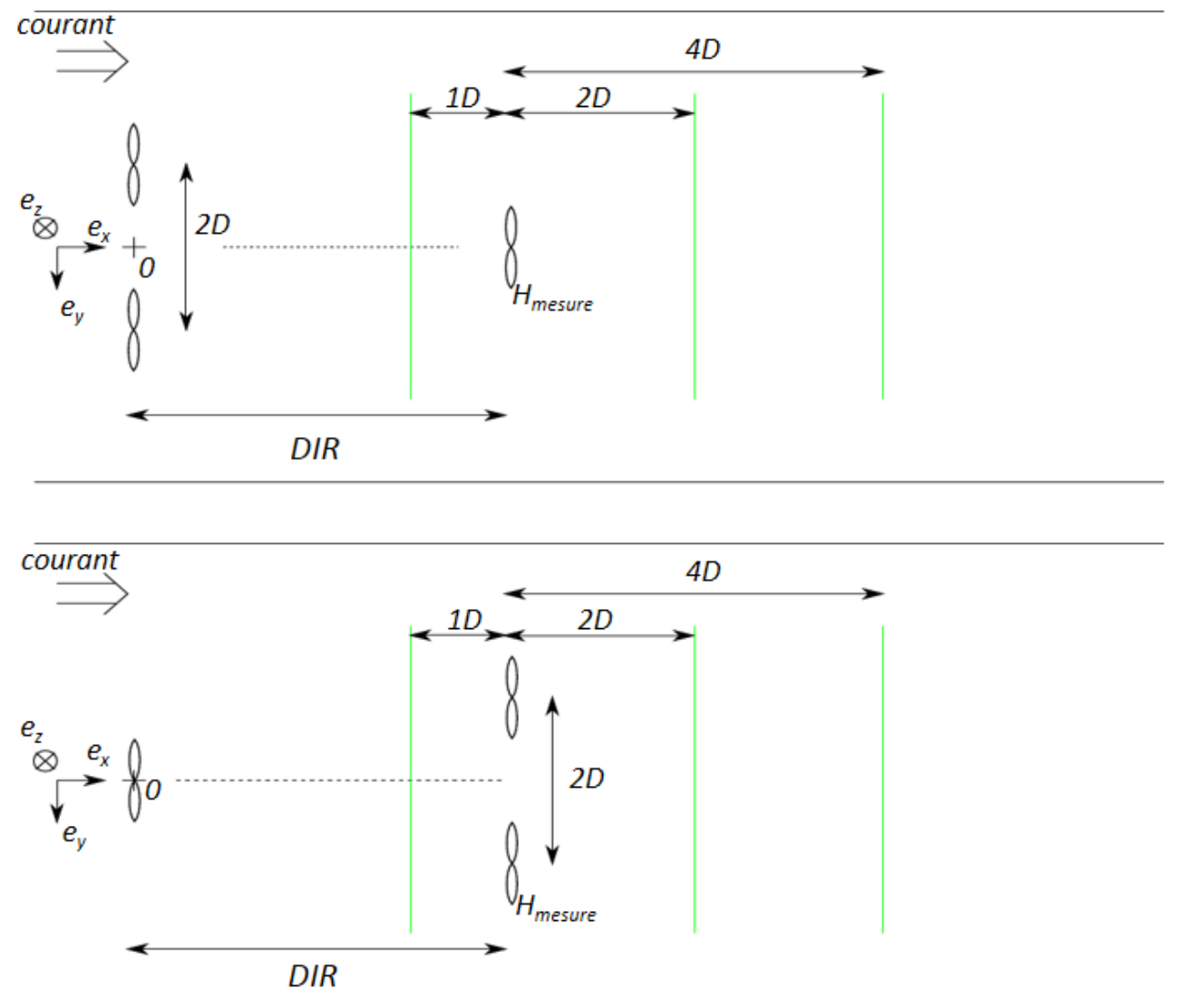

Figure 2. Vue de dessus de la configuration 1 (en haut) et de la configuration 2 (en bas) avec la distance inter-rangées (DIR) valant 4 diamètres ou 6 diamètres. Les traits verts représentent les positons des profils de mesures laser. 


\section{Thème 5 - Énergies et ressources marines}

Les mesures ont été menées à une seule vitesse de courant : $0.8 \mathrm{~m} / \mathrm{s}$. A cette vitesse, les pales sont conçues pour être utilisées à des TSR entre 0 et 7, avec un maximum pour le coefficient de puissance autour de $T S R=4$ (CARLIER et al., 2014). C'est également à cette vitesse de rotation que les résultats sont principalement présentés dans la suite. Les mesures de sillage sont réalisées à l'aide d'un système de Vélocimétrie Laser Doppler (LDV) à 2 composantes. Deux taux de turbulence de l'écoulement amont ont été considérés dans cette étude : $I_{\infty}=3$ et $15 \%$. Ces deux niveaux de turbulence sont obtenus directement à partir de la génération du courant dans le bassin, avec et sans nid d'abeilles, respectivement (MYCEK et al., 2014).

Trois machines tournant dans le même sens de rotation étant utilisées, deux configurations ont été retenues. La première repose sur l'idée que la vitesse du courant augmente entre deux machines côte à côte face au courant. De ce fait, le positionnement de la configuration 1 consiste en deux machines côte à côte (espacées de 2D) placées en amont et une machine en aval centrée dans le bassin (figure 2, en haut) pour profiter de cette "sur-vitesse" potentielle. Deux espacements ont été testés pour cette configuration 1 : 4 puis 6 diamètres entre la première et la seconde rangées d'hydroliennes (distance inter-rangées, DIR). Les profils laser ont été effectués à $1 D$ en amont de la seconde rangée et à $2 D$ et $4 D$ en aval.

La configuration 2 est symétrique à la précédente avec une unique machine en amont et deux machines en aval (figure 2 en bas), les écartements et profils LDV étudiés étant identiques à ceux de la configuration 1.

\section{Effets d'interaction et mise en évidence du rôle de la turbulence ambiante}

\subsection{Configuration 1}

La figure 3 présente les résultats obtenus sur l'hydrolienne avale (nommée $H_{\text {mesure }}$ sur la figure 2) en termes de performances lorsque les trois hydroliennes se trouvent dans la configuration 1, pour des espacements inter-rangées de $4 D$ et $6 D$ et pour $I_{\infty}$ égal à $3 \%$ et 15\%. Pour un taux d'intensité turbulente donné, les courbes de performance de l’hydrolienne avale ne présentent que de faibles différences entre les deux espacements inter-rangées : la montée en puissance se fait jusqu'à une valeur de TSR de 4 (maximum de $C_{P}$ ) puis la descente se fait plus lentement jusqu'à un TSR de 7 , tandis que la montée en $C_{T}$ est plus régulière. Bien que la forme globale des courbes reste similaire, l'impact du taux d'intensité turbulente sur les performances de l'hydrolienne est, par contre, bien plus significatif : environ $30 \%$ de diminution de $C_{P}$ pour un $T S R=4$ avec un taux de turbulence $I_{\infty}=15 \%$.

La figure 4 présente les résultats obtenus sur l'hydrolienne avale en termes de développement de sillage lorsque les trois hydroliennes se trouvent dans la configuration 1 à $T S R=4$, pour des espacements inter-rangées de $4 D$ et $6 D$ et pour des 


\section{XIII ${ }^{\text {èmes }}$ Journées Nationales Génie Côtier - Génie Civil \\ Dunkerque, 2-4 juillet 2014}

taux de turbulence $I_{\infty}$ de $3 \%$ et $15 \%$. Pour les deux espacements inter-rangées considérés, les différences ne sont pas significatives, sauf pour le profil amont de la seconde rangée $(-1 D)$ où les vitesses sont moins atténuées dans la configuration avec un espacement de $6 D$.

Il est intéressant de constater que, contrairement à toute attente, la vitesse n'augmente pas entre les deux hydroliennes avales, puisque le ratio des vitesses atteint 1 pour les deux écartements considérés, pour $I_{\infty}=3 \%$ et à $y / D=0$ (profil à $-1 D$ ). Cette vitesse diminue même significativement d'environ 10 à $15 \%$ pour le même profil, au même endroit pour $I_{\infty}=15 \%$ (raison pour laquelle la courbe correspondante du $C_{P}$ est inférieure).

L'impact du taux de turbulence ambiante sur le développement des sillages est donc très important : les profils sont plus "lissés" avec des atténuations de vitesse beaucoup plus faibles. En effet, alors que celle-ci peut atteindre 45 à $70 \%$ derrière la première rangée de machine (profil à $-1 D$ ) pour $I_{\infty}=3 \%$, elle se limite à des valeurs comprises entre 10 et $30 \%$ pour $I_{\infty}=15 \%$.
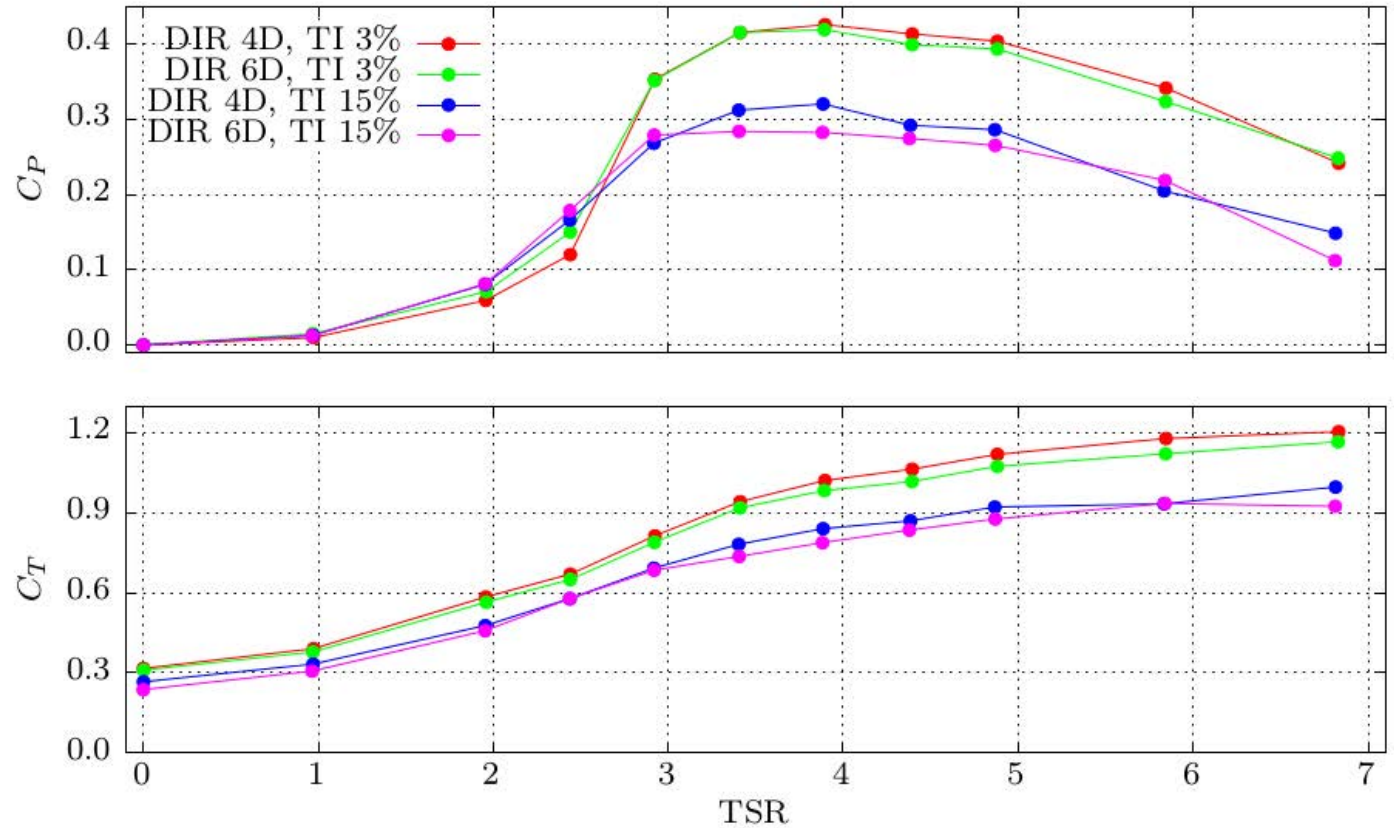

Figure 3. Performance de l'hydrolienne avale $\left(H_{\text {mesure }}\right.$ sur la figure 2$)$ pour différentes distances inter-rangées (DIR) et différents taux de turbulence incident $\left(I_{\infty}\right)$, dans la configuration 1 (les deux autres machines sont à TSR=4). 

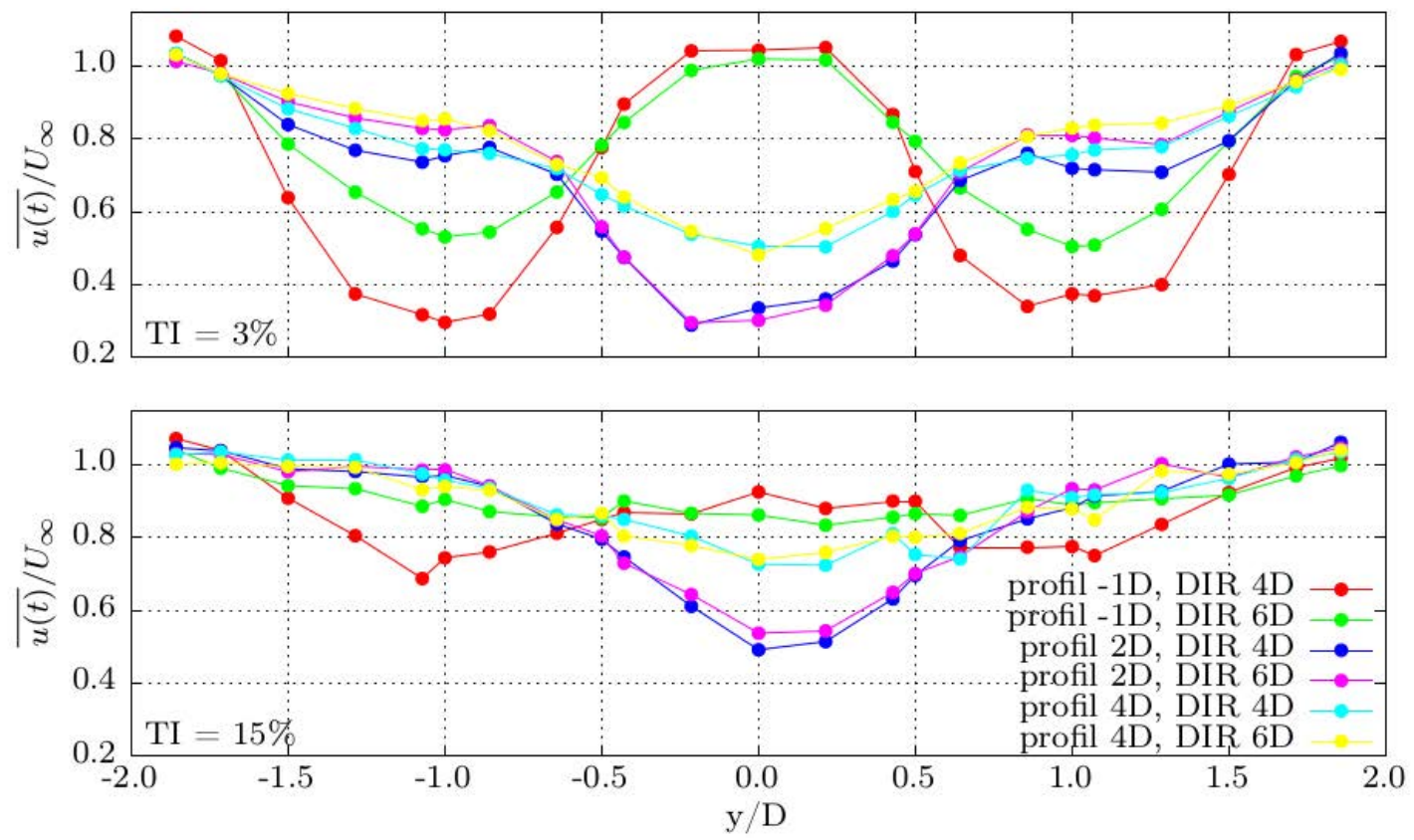

Figure 4. Profils de vitesse moyenne pour différentes distances inter-rangées (DIR) et différents taux de turbulence incident $\left(I_{\infty}\right)$, dans la configuration 1 (toutes les machines sont à TSR=4).

\subsection{Configuration 2}

La figure 5 présente les résultats obtenus sur l'hydrolienne instrumentée $H_{\text {mesure }}$ en terme de performance lorsque les trois hydroliennes se trouvent dans la configuration 2 (figure 1 en bas), pour des espacements inter-rangées de $4 D$ et $6 D$ et pour des taux de turbulence $I_{\infty}$ de $3 \%$ et $15 \%$. Les mêmes conclusions que pour la configuration 1 peuvent être établies: l'espacement inter-rangées n'engendre pas de différences significatives sur les performances de l'hydrolienne en aval, contrairement au taux de turbulence ambiant. Une différence d'environ $10 \%$ est observée à partir de $T S R=3$ entre les deux taux de turbulence sur les $C_{P}$. D'autre part, l'hydrolienne instrumentée $H_{\text {mesure, }}$ dans cette configuration 2, présente des performances plus élevées que dans la configuration 1 , en particulier pour le taux de turbulence $I_{\infty}=15 \%$.

La figure 6 présente les résultats obtenus en termes de développement de sillage lorsque les trois hydroliennes se trouvent dans la même configuration 2. De la même manière que pour la configuration 1 , les différences ne sont pas significatives entre les deux espacements inter-rangées, sauf pour les profils amonts de la seconde rangée $(-1 D)$ où les vitesses sont moins atténuées pour celui de $6 D$ que celui de $4 D$, autour de $y / D=0$. L'impact du taux de turbulence ambiant sur le développement des sillages est quant à lui également très important : avec $I_{\infty}=15 \%$, les atténuations de vitesses sont de l'ordre de $40 \%$ sur les profils à $2 D$, alors qu'elles atteignent $60 \%$ sur les mêmes profils à $I_{\infty}=3 \%$. 


\section{XIII ${ }^{\text {èmes }}$ Journées Nationales Génie Côtier - Génie Civil \\ Dunkerque, 2-4 juillet 2014}
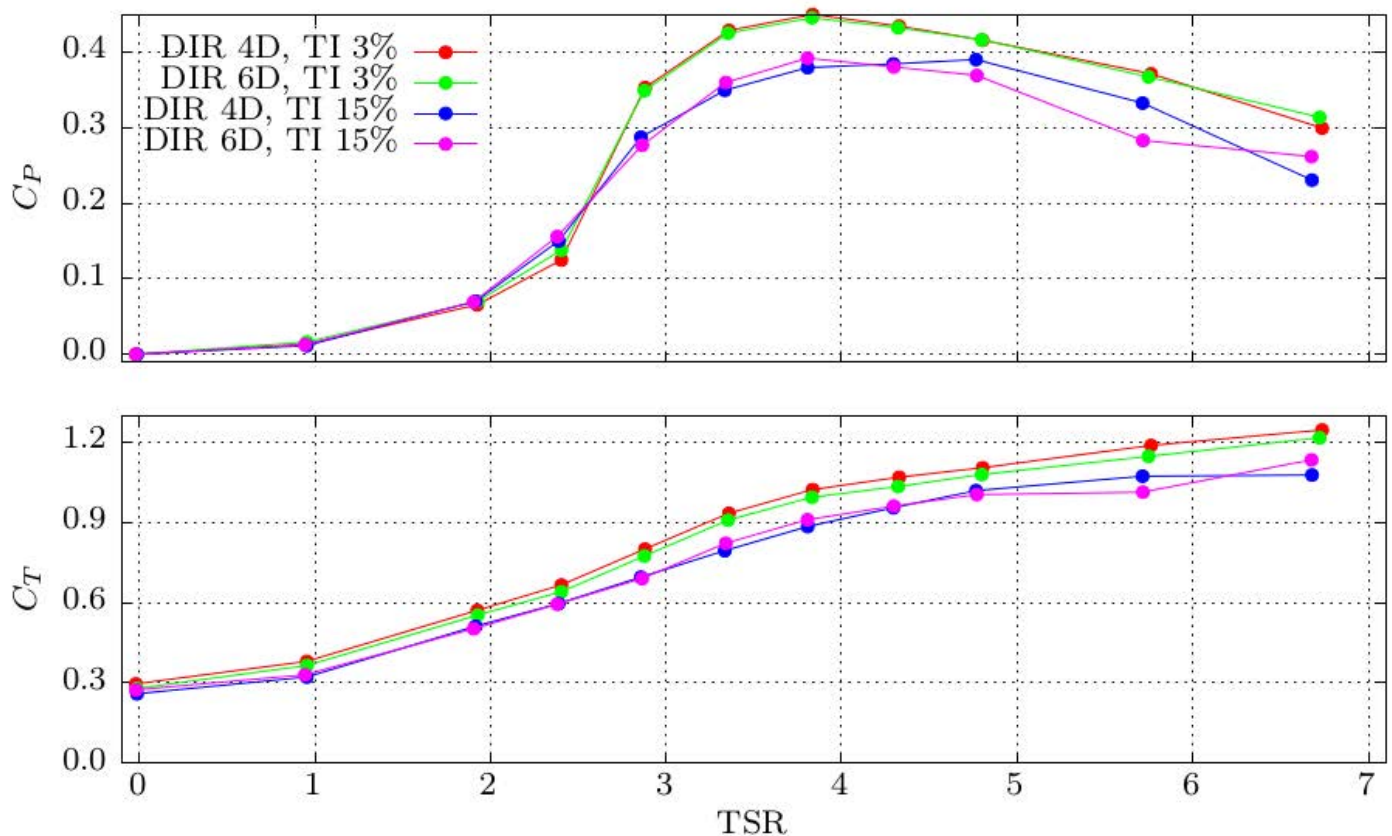

Figure 5. Performance de l'hydrolienne instrumentée ( $H_{\text {mesure }}$ sur la figure 2$)$ pour différentes distances inter-rangées (DIR) et différents taux de turbulence incident $\left(I_{\infty}\right)$, dans la configuration 2 (les autres machines sont à TSR=4).
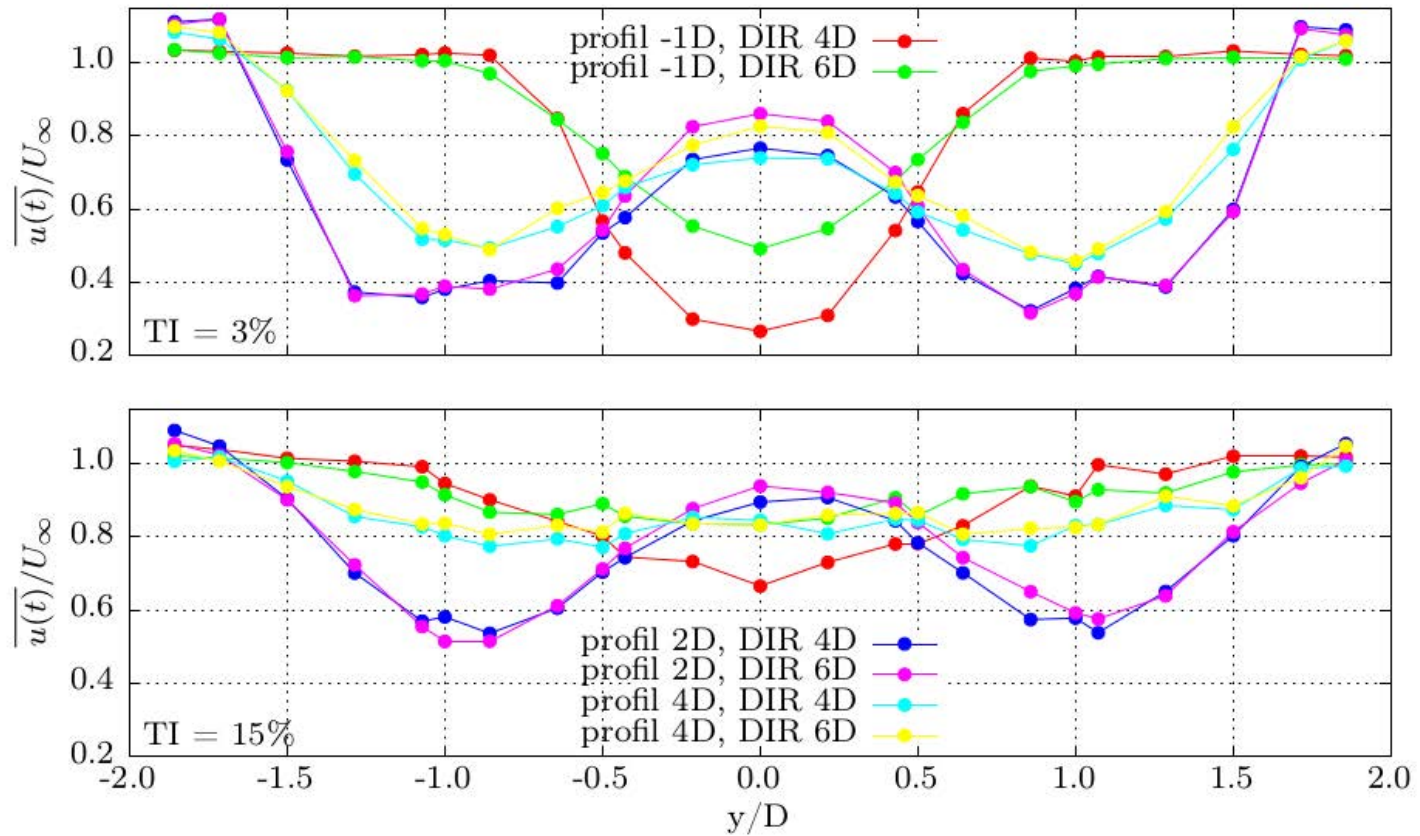

Figure 6. Profils de vitesse moyenne pour différentes distances inter-rangées (DIR) et différents taux de turbulence incident $\left(I_{\infty}\right)$, dans la configuration 2 (toutes les machines sont à TSR=4). 


\section{Conclusion}

Les résultats obtenus pour les deux configurations montrent le rôle évident du taux de turbulence ambiante sur les performances des machines et sur le développement de sillages, et ce quel que soient les espacements inter-rangées.

On peut en effet noter une diminution globale des coefficients de puissance et de traînée lorsque le taux de turbulence augmente (avec une diminution moyenne de 30\% pour la configuration 1 et de $10 \%$ pour la configuration 2), ainsi qu'un développement de sillage très vite limité avec des atténuations de vitesse très faibles à seulement quelques diamètres en aval des hydroliennes.

Cette conclusion est d'autant plus importante que l'installation des futures hydroliennes en mer se fera dans des zones où les courants sont très énergétiques et où les taux de turbulence ambiante sont très importants (entre 15 et 25\% environ pour le Raz Blanchard, selon estimations). Les modèles numériques visant à caractériser la ressource disponible d'un site hydrolien et surtout la production d'une future ferme d'hydroliennes, devront donc prendre en compte ce paramètre non négligeable et adapter leur modèle de fermeture turbulente (ou calculer directement cette turbulence le cas échéant), notamment pour l'estimation des effets d'interaction entre machines. Cette étude devrait constituer une base de données non négligeable pour la validation de futures modélisations numériques.

\section{Références bibliographiques}

BATIFOULIER F., KERVELLA Y., LABORBE R., CUNY J., CAILLAUD M., LAZURE P., CAYOCCA F. (2012). Impact hydrodynamique de la mise en place d'un parc d'hydroliennes en Baie du Mont $S^{t}$-Michel. Journées Nationales Génie Côtier Génie Civil, Cherbourg, pp 809-818. http://dx.doi.org/10.5150/jngcgc.2012.088-B

CARLIER C., MYCEK P, GAURIER B., GERMAIN G., PINON G., RIVOALEN E. (2014). Etude expérimentale et numérique du comportement d'hydroliennes à axe horizontal. Journées Nationales Génie Côtier - Génie Civil, Dunkerque. http://dx.doi.org/10.5150/jngcgc.2014.079

MYCEK P., GAURIER B., GERMAIN G., PINON G. RIVOALEN E. (2014). Experimental study of the turbulence intensity effects on marine current turbines behavior. Part I: one single turbine. Renewable Energy, Vol. 66, pp 729-746. http://dx.doi.org/10.1016/j.renene.2013.12.036

MYERS L. BAHAJ A. (2010). Experimental analysis of the flow field around horizontal axis tidal turbines by use of scale mesh disk rotor simulators. Ocean Engineering, Vol. 37, pp 218-227. http://dx.doi.org/10.1016/j.oceaneng.2009.11.004 